\title{
Produção e qualidade de frutos de pessegueiros 'Chimarrita' e 'Maciel' sobre diferentes porta-enxertos
}

\author{
Simone Padilha Galarça(1), José Carlos Fachinello(1), Débora Leitzke Betemps ${ }^{(1)}$, \\ Nicácia Portella Machado(1), Luciane Both Haas ${ }^{(1)}$, Marcos Ernani Prezotto ${ }^{(1)}$ e Andressa Comiotto ${ }^{(2)}$
}

(1)Universidade Federal de Pelotas, Departamento de Fitotecnia, Caixa Postal 354, CEP 96010-900 Pelotas, RS. E mail: sgalarca@superig.com.br, jfachi@ufpel.tche.br, deborabetemps@hotmail.com, nicacia@gmail.com, luciane.haas@yahoo.com.br, marcosprezotto@hotmail.com (2)Instituto Federal Rio Grande do Sul, Avenida Osvaldo Aranha, no 540, Bairro Juventude da Enologia, CEP 95700-000 Bento Gonçalves, RS. E-mail: andressa.comiotto@bento.ifrs.edu.br

Resumo - O objetivo deste trabalho foi avaliar a influência de porta-enxertos sobre a qualidade pós-colheita dos frutos e produção das cultivares de pessegueiro Chimarrita e Maciel. O experimento foi realizado nos municípios de Bento Gonçalves, Eldorado do Sul e Capão do Leão, no Estado do Rio Grande do Sul, durante três anos. Foram avaliados os porta-enxertos 'Aldrighi', 'Capdeboscq', 'Flordaguard', 'Nemaguard', 'Okinawa' e Umezeiro. As plantas foram conduzidas no sistema em "V", no espaçamento 1,5x5,0 m. As seguintes variáveis foram avaliadas: diâmetro e massa de frutos, número de frutos por planta, produção por planta, eficiência produtiva, firmeza de polpa e conteúdo de sólidos solúveis. As variáveis avaliadas foram pouco influenciadas pelos porta-enxertos, na média de produção dos três anos. A cultivar Maciel apresentou alta eficiência produtiva em todos os porta-enxertos. O porta-enxerto 'Nemaguard' proporcionou maior produção à cultivar Maciel em Bento Gonçalves, enquanto o porta-enxerto Umezeiro, a menor produção na cultivar Chimarrita em Eldorado do Sul. A firmeza de polpa foi a variável de qualidade de frutos mais influenciada pelos porta-enxertos, conforme as condições do local.

Termos para indexação: Prunus, eficiência produtiva, firmeza de polpa, pós-colheita, sólidos solúveis.

\section{Yield and fruit quality of 'Chimarrita' and 'Maciel' peaches on different rootstocks}

\begin{abstract}
The objective of this work was to evaluate the influence of rootstocks on fruit postharvest quality and productivity of the peach cultivars 'Chimarrita' and 'Maciel'. The experiment was carried out in the Municipalities of Bento Gonçalves, Eldorado do Sul, and Capão do Leão, in the state of Rio Grande do Sul, Brazil, during three years. The rootstocks 'Aldrighi', 'Capdeboscq', 'Flordaguard', 'Nemaguard', 'Okinawa', and 'Mume' were evaluated. The plants were conducted using the "V" system, with $1.5 \times 5.0 \mathrm{~m}$ spacing. The following variables were evaluated: fruit mass and diameter, number of fruit per plant, yield per plant, production efficiency, pulp firmness, and contents of soluble solids. The analyzed variables were little influenced by rootstocks, on the three-year production average. Cultivar Maciel had high-production efficiency in all rootstocks. The rootstock 'Nemaguard' provided the highest yield to cultivar Maciel in Bento Gonçalves, while the rootstock Mume, the lowest production to 'Chimarrita', in Eldorado do Sul. Firmness was the fruit quality variable most influenced by the rootstocks, depending on local conditions.
\end{abstract}

Index terms: Prunus, production efficiency, pulp firmness, postharvest, soluble solids.

\section{Introdução}

O sul do Rio Grande do Sul tem como polo de produção de pêssegos o Município de Pelotas, em que mais de $90 \%$ da produção é destinada ao processamento industrial (Timm et al., 2007). Em municípios da região de Porto Alegre, a produção é destinada ao consumo in natura. Na Encosta Superior do nordeste do estado, dez municípios, entre eles Bento Gonçalves, produzem variedades de polpa branca, para consumo in natura, e polpa amarela de duplo propósito (Protas \& Madail, 2003).

Apesar de o Rio Grande do Sul ser responsável por $65,1 \%$ dos pêssegos produzidos no Brasil (Fachinello et al., 2011), sua produtividade média é baixa $\left(8,95 \mathrm{Mg} \mathrm{ha}^{-1}\right)$, se comparada à média nacional $\left(10,93 \mathrm{Mg} \mathrm{ha}^{-1}\right)$ e aos demais estados produtores: Santa Catarina (12,36 Mg ha $\left.{ }^{-1}\right)$, Paraná (10,22 $\left.\mathrm{Mg} \mathrm{ha}^{-1}\right)$ e São Paulo (21,06 $\mathrm{Mg} \mathrm{ha}^{-1}$ ) (Instituto Brasileiro de Geografia e Estatística, 2012). Para melhorar a produção, 
aspectos de manejo, como irrigação, adubação, controle fitossanitário e uso de mudas fiscalizadas, são importantes. Além disso, o desenvolvimento e avaliação de cultivares de porta-enxerto são fundamentais.

Os porta-enxertos influenciam a adaptação climática da cultivar-copa, assim como as diferentes respostas fisiológicas a condições adversas, como temperaturas elevadasduranteafloração, deficithídricoevariabilidade das temperaturas hibernais, o que afeta a formação dos gametas sexuais e produz variação no padrão produtivo das cultivares, de acordo com os anos de cultivo (Nava et al., 2009). Assim, é importante que os produtores e a assistência técnica tenham informações específicas sobre os atributos e as limitações dos porta-enxertos, e sobre seu comportamento em diferentes solos e condições de clima (Rato et al., 2008).

Este trabalho basea-se na premissa de que há interação entre porta-enxertos, cultivares-copa e locais de cultivo, quanto à produção e qualidade dos frutos (Rossi et al., 2004; Rocha et al., 2007; Picolotto et al., 2009; Comiotto et al., 2012).

O objetivo deste trabalho foi avaliar a influência de porta-enxertos sobre a qualidade pós-colheita dos frutos e produtividade das cultivares de pessegueiro Chimarrita e Maciel.

\section{Material e Métodos}

O experimento foi realizado nos municípios de Bento Gonçalves, Eldorado do Sul e Capão do Leão, no Estado do Rio Grande do Sul. Em Bento Gonçalves, utilizou-se o campo experimental da Embrapa Uva e Vinho $\left(29^{\circ} 09^{\prime} 44^{\prime \prime S}, 51^{\circ} 31^{\prime} 50^{\prime \prime} \mathrm{W}\right.$, à altitude de $\left.640 \mathrm{~m}\right)$. O clima local é classificado como $\mathrm{Cfb}$ (temperado, úmido e com verão ameno), de acordo com Köppen, e o solo é classificado como Chernossolo (Streck et al., 2008). A área do experimento tem problemas de drenagem, o solo é argiloso, com pontos de acúmulo de água.

Em Eldorado do Sul, utilizou-se a Estação Experimental Agronômica da Universidade Federal do Rio Grande do Sul (29 57'16"S, 51³7131"W, à altitude de $30 \mathrm{~m}$ ). O clima da região é Cfa (temperado úmido, com verão quente), de acordo com Köppen, e o solo é classificado como Argissolo Vermelho distrófico típico (Streck et al., 2008). A área do experimento é de replantio, com permanência de pomar de pessegueiro por 20 anos e pousio de três anos, antes da instalação do experimento.

Em Capão do Leão, utilizou-se o Centro Agropecuário da Palma, pertencente à Universidade Federal de Pelotas $\left(31^{\circ} 52^{\prime} 00^{\prime \prime} \mathrm{S}, 52^{\circ} 21^{\prime} 24^{\prime \prime} \mathrm{W}\right.$, à altitude de $\left.13,24 \mathrm{~m}\right)$. O clima é Cfa, de acordo com Köppen, e o solo é classificado como Argissolo Vermelho-Amarelo distrófico (Streck et al., 2008).

Os dados de temperatura, precipitação e insolação média mensal de três anos, para as localidades de Bento Gonçalves, Eldorado do Sul e Capão do Leão, foram obtidos nas estações meteorológicas existentes nas estações experimentais (Tabela 1). As características físico-químicas dos solos das áreas experimentais estão apresentadas na Tabela 2 .

As cultivares-copa utilizadas foram: Chimarrita (polpa branca, consumo in natura) e Maciel (polpa amarela, duplo propósito), ambas Prunus persica (L.) Batsch. Essas cultivares foram enxertadas sobre os porta-enxertos: P. persica, 'Aldrighi', 'Capdeboscq' e 'Okinawa'; P. persica x P. davidiana Carr., 'Flordaguard' e 'Nemaguard'; e P. mume Sieb \& Zucc, Umezeiro.

Os campos experimentais foram manejados de acordo com as normas de produção integrada para pêssego

Tabela 1. Temperatura, precipitação e insolação média mensal de três anos (2009, 2010 e 2011), em Bento Gonçalves, Eldorado do Sul e Capão do Leão, no Rio Grande do Sul.

\begin{tabular}{lccc}
\hline Local & $\begin{array}{c}\text { Temperatura } \\
\left({ }^{\circ} \mathrm{C}\right)\end{array}$ & $\begin{array}{c}\text { Precipitação } \\
(\mathrm{mm})\end{array}$ & $\begin{array}{c}\text { Radiação global } \\
\left(\mathrm{cal} \mathrm{cm}^{-2} \text { por dia }\right)\end{array}$ \\
\hline Bento Gonçalves, RS & 18,5 & 201,22 & $-^{(1)}$ \\
Eldorado do Sul, RS & 17,3 & 175,5 & 366,2 \\
Capão do Leão, RS & 15,5 & 123,11 & 331,3 \\
\hline
\end{tabular}

(1)Não disponível.

Tabela 2. Resultados da análise do solo dos diferentes locais de cultivo utilizados.

\begin{tabular}{|c|c|c|c|c|c|c|c|c|c|c|c|c|}
\hline \multirow[t]{2}{*}{ Local } & \multirow[t]{2}{*}{$\mathrm{pH}$} & MO & P (Mehlich) & $\mathrm{K}$ & $\mathrm{Ca}$ & $\mathrm{Mg}$ & $\mathrm{H}+\mathrm{Al}$ & \multirow{2}{*}{$\begin{array}{l}\text { SB } \\
(\%)\end{array}$} & $\mathrm{Na}$ & $\mathrm{Cu}$ & $\mathrm{Zn}$ & \multirow[t]{2}{*}{$\mathrm{Mn}$} \\
\hline & & $(\%)$ & \multicolumn{2}{|c|}{-----( $\left(\mathrm{mg} \mathrm{dm}^{-3}\right)$------ } & \multicolumn{3}{|c|}{------- $\left(\mathrm{cmol}_{\mathrm{c}} \mathrm{dm}^{-3}\right)$-------- } & & ---- & $---(m$ & $-3)---$ & \\
\hline Bento Gonçalves, RS & 5,9 & 3,9 & 35 & 330 & 8,8 & 2,8 & 3,5 & 78 & 12 & 30,1 & 16,7 & 17 \\
\hline Eldorado do Sul, RS & 5,8 & 1,9 & 8,3 & 81 & 2,4 & 0,9 & 2,5 & 58 & 3 & 6,3 & 1,8 & 14 \\
\hline Capão do Leão, RS & 5,4 & 1,8 & 27,3 & 153 & 7 & 0,8 & 3,1 & 49 & 7 & 4,5 & 5,7 & 48 \\
\hline
\end{tabular}

MO, matéria orgânica; SB, saturação por bases. 
(Fachinello, 2003). Os pomares foram instalados em julho de 2006 e avaliados nos anos de 2009, 2010 e 2011 (3을 4o e 5o anos após a implantação). Utilizou-se o sistema de condução em "V", com espaçamento de $5 \mathrm{~m}$ entre linhas e de $1,5 \mathrm{~m}$ entre plantas.

Utilizou-se o delineamento experimental de blocos ao acaso, com três repetições e cinco plantas por parcela, em que as três plantas centrais na linha de plantio foram consideradas unidades de observação, e as demais como bordadura. Empregou-se o arranjo unifatorial (porta-enxertos), para cada cultivar-copa e cada local de cultivo, na média de três anos. Porém, nas avaliações de qualidade pós-colheita, foram utilizados 60 frutos por bloco de cada unidade experimental.

As variáveis de produção analisadas foram: diâmetro dos frutos (mm), medido com paquímetro digital; massa dos frutos $(\mathrm{g})$; número de frutos por planta; produção por planta, determinada a partir da multiplicação da massa dos frutos pelo número de frutos por planta $(\mathrm{kg})$; e eficiência produtiva, obtida com base nas médias de produção e volume de copa $\left(\mathrm{kg} \mathrm{m}^{-3}\right)$.

As variáveis de qualidade pós-colheita dos frutos avaliadas foram: firmeza de polpa $(\mathrm{N})$, avaliada na região equatorial dos frutos, em lados opostos, com retirada da casca nestes locais, determinada com uso do penetrômetro digital TR (Turoni-Italy, modelo 53205), com ponteira de $8 \mathrm{~mm}$; e sólidos solúveis totais ( ${ }^{\circ}$ Brix), determinado com refratômetro digital PAL-1 (Atago, Tóquio, Japão), com correção automática da temperatura. As avaliações para a cultivar Chimarrita foram feitas no início de dezembro e, para a cultivar Maciel, no final de dezembro.

Todas as variáveis foram analisadas nos três locais, nas duas cultivares, durante os três anos, com exceção de 'Chimarrita' sobre Umezeiro, na localidade de Eldorado do Sul, cujas variáveis foram avaliadas em apenas um ciclo (2010), porque esta cultivar não produziu nos outros anos. Foi avaliada a média dos três anos, para todas as variáveis. Para verificação dos efeitos do fator porta-enxerto sobre as copas, nos diferentes locais, realizou-se a análise de variância. Realizou-se o agrupamento de médias univariado, pelo teste de Scott-Knott, a 5\% de probabilidade, conforme Cruz et al. (2004), com utilização do programa estatístico Genes (Cruz, 2001).

\section{Resultados e Discussão}

As temperaturas médias dos três anos, em Bento Gonçalves, foram maiores do que as de Eldorado do Sul e Capão do Leão (Tabela 1). O solo em Bento Gonçalves apresentou maior teor de matéria orgânica, fósforo, potássio e cálcio (Tabela 2).

Em Bento Gonçalves, não houve influência dos porta-enxertos sobre as variáveis analisadas, na cultivar Chimarrita. Na cultivar Maciel, os porta-enxertos afetaram a produção estimada, e o porta-enxerto 'Nemaguard' proporcionou maior valor desta variável (Tabela 3). Estes resultados corroboram os obtidos por Blažková et al. (2010), em que a cultivar de porta-enxerto e o volume de copa influenciaram a

Tabela 3. Produção e qualidade pós-colheita dos pessegueiros 'Chimarrita' e 'Maciel', sobre diferentes porta-enxertos, em Bento Gonçalves, $\operatorname{RS}^{(1)}$.

\begin{tabular}{|c|c|c|c|c|c|c|c|}
\hline Porta-enxerto & $\begin{array}{c}\text { Diâmetro } \\
\text { de frutos }(\mathrm{mm})\end{array}$ & $\begin{array}{c}\text { Massa } \\
\text { de frutos }(\mathrm{g})\end{array}$ & $\begin{array}{l}\text { Número } \\
\text { de frutos }\end{array}$ & $\begin{array}{l}\text { Produção por } \\
\text { planta }(\mathrm{kg})\end{array}$ & $\begin{array}{c}\text { Eficiência } \\
\text { produtiva }\left(\mathrm{kg} \mathrm{m}^{-3}\right)\end{array}$ & $\begin{array}{c}\text { Firmeza } \\
\text { de polpa }(\mathrm{N})\end{array}$ & $\begin{array}{c}\text { Sólidos } \\
\text { solúveis ( }{ }^{\circ} \text { Brix) }\end{array}$ \\
\hline & \multicolumn{7}{|c|}{ 'Chimarrita' } \\
\hline 'Aldrighi' & $54,5^{\mathrm{ns}}$ & $90,6^{\mathrm{ns}}$ & $68,0 \mathrm{~ns}$ & $5,7^{\mathrm{ns}}$ & $5,1^{\mathrm{ns}}$ & $30,0^{\mathrm{ns}}$ & $11,9^{\mathrm{ns}}$ \\
\hline 'Capdeboscq' & 51,9 & 80,5 & 74,0 & 5,3 & 4,1 & 23,1 & 12,4 \\
\hline 'Flordaguard' & 48,9 & 79,2 & 83,0 & 5,7 & 4,7 & 21,4 & 13,1 \\
\hline 'Nemaguard' & 50,9 & 80,4 & 93,0 & 7,9 & 5,7 & 31,2 & 12,4 \\
\hline 'Okinawa' & 49,7 & 78,5 & 96,0 & 7,4 & 6,9 & 25,8 & 12,7 \\
\hline Umezeiro & 53,6 & 86,2 & 35,0 & 2,9 & 5,0 & 26,2 & 12,4 \\
\hline \multirow[t]{2}{*}{ CV (\%) } & 7,0 & 17,8 & 19,8 & 34,0 & 19,9 & 14,8 & 6,5 \\
\hline & \multicolumn{7}{|c|}{ 'Maciel' } \\
\hline 'Aldrighi' & $58,8^{\mathrm{ns}}$ & $114,0^{\mathrm{ns}}$ & $65,0^{\mathrm{ns}}$ & $6,8 b$ & $4,4^{\mathrm{ns}}$ & $26,0^{\mathrm{ns}}$ & $13,8^{\mathrm{ns}}$ \\
\hline 'Capdeboscq' & 59,0 & 112,9 & 64,0 & $6,8 b$ & 4,7 & 28,4 & 13,7 \\
\hline 'Flordaguard' & 51,6 & 92,1 & 39,0 & $3,5 b$ & 2,7 & 29,5 & 14,8 \\
\hline 'Nemaguard' & 58,6 & 119,5 & 72,0 & $12,2 \mathrm{a}$ & 4,1 & 27,5 & 14,3 \\
\hline 'Okinawa' & 56,4 & 103,5 & 47,0 & $4,4 \mathrm{~b}$ & 2,9 & 32,8 & 14,5 \\
\hline Umezeiro & 60,0 & 120,0 & 41,0 & $5,5 b$ & 4,4 & 31,5 & 13,3 \\
\hline$\overline{C V}(\%)$ & 5,4 & 11,6 & 21,8 & 25,4 & 40,0 & 7,6 & 5,4 \\
\hline
\end{tabular}

${ }^{(1)}$ Médias (três anos de cultivo) seguidas de letras iguais não diferem, entre si, pelo teste de Scott-Knott, a 5\% de probabilidade. ${ }^{\text {ns }}$ Não significativo. 
produção por planta, sendo que os porta-enxertos mais vigorosos proporcionaram maior volume de copa, com consequente aumento na produção. O porta-enxerto 'Nemaguard' é considerado um dos mais vigorosos (Medeiros \& Raseira, 1998) e, possivelmente por isso, proporcionou o melhor resultado em Bento Gonçalves, pois o solo dessa localidade tinha problemas de drenagem e plantas mais vigorosas conseguem melhor desenvolvimento nestas condições.

Em Eldorado do Sul, apenas a produção da cultivarcopa Chimarrita foi influenciada pelos porta-enxertos. O porta-enxerto Umezeiro proporcionou a menor produção (Tabela 4). Esse resultado possivelmente foi decorrência da menor capacidade de armazenamento de carboidratos e da baixa fixação de carbono por plantas enxertadas em porta-enxertos ananizantes, como o Umezeiro (Gonçalves et al., 2006). Essas características reduzem o crescimento vegetativo.

Em Capão do Leão, a firmeza de polpa foi influenciada pelos porta-enxertos, em ambas as cultivares-copa. $\mathrm{Na}$ cultivar Chimarrita, o porta-enxerto Umezeiro proporcionou menor firmeza aos frutos, e 'Aldrighi', 'Capdeboscq' e 'Nemaguard', maior. Nessa localidade, a cultivar Maciel apresentou maior eficiência produtiva quando enxertada sobre o Umezeiro, e maior firmeza quando sobre 'Aldrighi' (Tabela 5).

Porta-enxertos que induzem menor porte e vigor às copas podem proporcionar maior eficiência produtiva às plantas, conforme observado por Cantuarias-Avilés et al. (2010). Segundo os autores, há relação linear negativa entre a eficiência produtiva e o volume de copa, até um determinado limite. A alta eficiência produtiva e o baixo vigor são importantes para sistemas de produção com alta densidade de plantio.

Hernández et al. (2010) observaram que plantas de damasqueiro, enxertadas sobre porta-enxertos de Prunus mais vigorosos, apresentavam maior produção e menor diâmetro e massa de frutos. Esses dados estão de acordo com os apresentados no presente trabalho quanto a produção por planta, mas discordam quanto as outras variáveis, que neste trabalho não foram influenciadas pelos porta-enxertos utilizados.

$\mathrm{Na}$ média de três anos, a produção foi pouco influenciada pelos porta-enxertos. No entanto, Rocha et al. (2007) encontraram influência significativa dos porta-enxertos sobre essa variável, no pessegueiro 'Chimarrita'. Os autores relatam, porém, que esta influência pode variar após os três primeiros anos da implantação do pomar. Picolotto et al. (2009) também observaram influência dos porta-enxertos sobre a produção da cultivar Chimarrita, no quarto, quinto e sexto anos após a implantação do pomar. Esses autores trabalharam com os dados de cada ano individualmente, o que tornou as análises mais sensíveis às variações ambientais.

Tabela 4. Produção e qualidade pós-colheita dos pessegueiros 'Chimarrita' e 'Maciel', sobre diferentes porta-enxertos, em Eldorado do Sul, $\mathrm{RS}^{(1)}$.

\begin{tabular}{|c|c|c|c|c|c|c|c|}
\hline Porta-enxerto & $\begin{array}{c}\text { Diâmetro } \\
\text { de frutos }(\mathrm{mm})\end{array}$ & $\begin{array}{c}\text { Massa } \\
\text { de frutos }(\mathrm{g}) \\
\end{array}$ & $\begin{array}{l}\text { Número } \\
\text { de frutos }\end{array}$ & $\begin{array}{c}\text { Produção por } \\
\text { planta }(\mathrm{kg})\end{array}$ & $\begin{array}{c}\text { Eficiência } \\
\text { produtiva }\left(\mathrm{kg} \mathrm{m}^{-3}\right)\end{array}$ & $\begin{array}{c}\text { Firmeza } \\
\text { de polpa }(\mathrm{N})\end{array}$ & $\begin{array}{c}\text { Sólidos } \\
\text { solúveis ( }{ }^{\circ} \text { Brix) } \\
\end{array}$ \\
\hline & \multicolumn{7}{|c|}{ 'Chimarrita' } \\
\hline 'Aldrighi' & $57,7^{\mathrm{ns}}$ & $109,3^{\text {ns }}$ & $34,0^{\text {ns }}$ & 4,9a & $1,4^{\mathrm{ns}}$ & $38,0^{\text {ns }}$ & $12,1 b$ \\
\hline 'Capdeboscq' & 73,9 & 105,4 & 35,0 & $5,1 \mathrm{a}$ & 1,4 & 36,8 & $11,9 \mathrm{~b}$ \\
\hline 'Flordaguard' & 58,3 & 108,5 & 42,0 & $4,8 \mathrm{a}$ & 1,8 & 36,6 & $11,5 b$ \\
\hline 'Nemaguard' & 57,6 & 109,3 & 35,0 & $4,0 \mathrm{a}$ & 2,2 & 37,3 & $12,2 \mathrm{~b}$ \\
\hline 'Okinawa' & 59,0 & 112,1 & 39,0 & $5,7 \mathrm{a}$ & 1,6 & 39,3 & $11,7 \mathrm{~b}$ \\
\hline Umezeiro $^{(2)}$ & 62,0 & 121,1 & 21,0 & $0,8 \mathrm{~b}$ & 0,6 & 34,8 & $13,6 \mathrm{a}$ \\
\hline \multirow[t]{2}{*}{ CV (\%) } & 18,4 & 5,9 & 23,8 & 31,3 & 46,0 & 9,8 & 4,7 \\
\hline & \multicolumn{7}{|c|}{ 'Maciel' } \\
\hline 'Aldrighi' & $61,4^{\mathrm{ns}}$ & $126,8^{\mathrm{ns}}$ & $56,0^{\text {ns }}$ & $6,7^{\mathrm{ns}}$ & $2,0^{\mathrm{ns}}$ & $29,0^{\text {ns }}$ & $13,0^{\text {ns }}$ \\
\hline 'Capdeboscq' & 60,2 & 117,9 & 77,0 & 8,5 & 2,4 & 27,1 & 12,6 \\
\hline 'Flordaguard' & 54,8 & 114,3 & 83,0 & 8,5 & 2,4 & 31,6 & 12,3 \\
\hline 'Nemaguard' & 61,4 & 122,8 & 64,0 & 7,7 & 2,3 & 33,5 & 12,5 \\
\hline 'Okinawa' & 60,9 & 121,7 & 53,0 & 5,9 & 1,7 & 34,6 & 13,1 \\
\hline Umezeiro & 60,2 & 120,5 & 52,0 & 6,0 & 2,6 & 35,8 & 12,4 \\
\hline $\mathrm{CV}(\%)$ & 5,2 & 5,2 & 12,9 & 27,1 & 34,7 & 9,8 & 3,0 \\
\hline
\end{tabular}

${ }^{(1)}$ Médias (três anos de cultivo) seguidas de letras iguais não diferem, entre si, pelo teste de Scott-Knott, a 5\% de probabilidade. ${ }^{(2)}$ Combinação avaliada apenas em 2011, por não ter proporcionado produção quantificável nos outros anos. n'Não significativo. 
Tabela 5. Produção e qualidade pós-colheita dos pessegueiros 'Chimarrita' e 'Maciel', sobre diferentes porta-enxertos, em Capão do Leão, $\mathrm{RS}^{(1)}$.

\begin{tabular}{|c|c|c|c|c|c|c|c|}
\hline Porta-enxerto & $\begin{array}{c}\text { Diâmetro } \\
\text { de frutos }(\mathrm{mm})\end{array}$ & $\begin{array}{c}\text { Massa } \\
\text { de frutos }(\mathrm{g})\end{array}$ & $\begin{array}{l}\text { Número } \\
\text { de frutos }\end{array}$ & $\begin{array}{l}\text { Produção por } \\
\text { planta }(\mathrm{kg})\end{array}$ & $\begin{array}{c}\text { Eficiência } \\
\text { produtiva }\left(\mathrm{kg} \mathrm{m}^{-3}\right)\end{array}$ & $\begin{array}{c}\text { Firmeza } \\
\text { de polpa }(\mathrm{N})\end{array}$ & $\begin{array}{c}\text { Sólidos } \\
\text { solúveis ( }{ }^{\circ} \text { Brix) }\end{array}$ \\
\hline & \multicolumn{7}{|c|}{ 'Chimarrita' } \\
\hline 'Aldrighi' & $57,0^{\mathrm{ns}}$ & $122,0^{\mathrm{ns}}$ & $104,0^{\mathrm{ns}}$ & $11,9^{\mathrm{ns}}$ & $2,9^{\text {ns }}$ & $41,3 \mathrm{a}$ & $10,7^{\mathrm{ns}}$ \\
\hline 'Capdeboscq' & 59,0 & 115,9 & 84,0 & 9,4 & 2,6 & $42,0 \mathrm{a}$ & 10,8 \\
\hline 'Flordaguard' & 54,6 & 109,5 & 138,0 & 13,2 & 3,0 & $36,3 b$ & 10,5 \\
\hline 'Nemaguard' & 59,4 & 124,8 & 103,0 & 12,3 & 3,3 & $44,0 \mathrm{a}$ & 10,6 \\
\hline 'Okinawa' & 58,4 & 114,9 & 89,0 & 9,5 & 3,0 & $35,3 b$ & 10,7 \\
\hline Umezeiro $^{(2)}$ & 57,5 & 121,7 & 64,0 & 7,9 & 3,9 & $29,4 \mathrm{c}$ & 10,5 \\
\hline \multirow[t]{2}{*}{ CV (\%) } & 4,7 & 6,2 & 7,8 & 18,4 & 16,6 & 5,9 & 3,5 \\
\hline & \multicolumn{7}{|c|}{ 'Maciel' } \\
\hline 'Aldrighi' & $62,0^{\text {ns }}$ & $143,0^{\text {ns }}$ & $113,0^{\text {ns }}$ & $14,8^{\mathrm{ns}}$ & $2,9 \mathrm{c}$ & $31,7 \mathrm{a}$ & $13,2^{\mathrm{ns}}$ \\
\hline 'Capdeboscq' & 66,2 & 146,2 & 125,0 & 17,2 & $3,8 b$ & $26,8 \mathrm{~b}$ & 13,1 \\
\hline 'Flordaguard' & 58,1 & 139,9 & 107,0 & 12,6 & $2,4 c$ & $28,8 \mathrm{~b}$ & 12,6 \\
\hline 'Nemaguard' & 67,7 & 149,1 & 131,0 & 17,6 & $3,4 \mathrm{c}$ & $26,8 \mathrm{~b}$ & 13,0 \\
\hline 'Okinawa' & 66,5 & 140,5 & 115,0 & 14,7 & $3,6 \mathrm{~b}$ & $28,2 b$ & 13,4 \\
\hline Umezeiro & 63,0 & 152,8 & 100,0 & 15,0 & $4,7 \mathrm{a}$ & $29,0 \mathrm{~b}$ & 13,2 \\
\hline CV (\%) & 5,4 & 4,1 & 8,2 & 24,5 & 12,3 & 4,3 & 3,1 \\
\hline
\end{tabular}

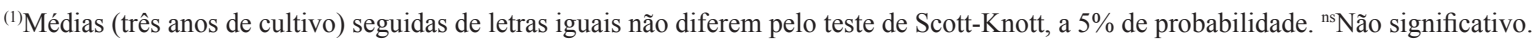

Observou-se maior número de frutos produzidos pelas combinações enxerto/porta-enxerto em Capão do Leão, indício de que essas combinações estão mais adaptadas às condições de clima e solo desta localidade. Em Bento Gonçalves, apesar da alta fertilidade natural do solo, problemas com a drenagem dificultaram o desenvolvimento das raízes e reduziram a produção.

\section{Conclusões}

1. A influência dos porta-enxertos sobre a produção por planta pode ser expressiva, mas varia de acordo com a cultivar-copa e o ambiente avaliado.

2. O porta-enxerto pode influenciar a eficiência produtiva, o conteúdo de sólidos solúveis e a firmeza de polpa das combinações copa/porta-enxerto utilizadas, mas esse efeito também varia de acordo com o ambiente e cultivar-copa.

3. A combinação de 'Chimarrita' com Umezeiro não é adequada em Eldorado do Sul.

\section{Agradecimentos}

Ao Conselho Nacional de Desenvolvimento Científico e Tecnológico (CNPq), pelo apoio financeiro e concessão de bolsa.

\section{Referências}

BLAŽKOVÁ, J.; DRAHOŠOVÁ, H.; HLUŠIČKOVÁ, I. Tree vigour, cropping, and phenology of sweet cherries in two systems of tree training on dwarf rootstocks. Horticultural Science, v.37, p.127-138, 2010.

CANTUARIAS-AVILÉS, T.; MOURÃO-FILHO, F. de A.A.; STUCHI, E.S.; SILVA, S.R.; ESPINOZA-NUÑEZ, E. Tree performance and fruit yield and quality of 'Okitsu' Satsuma mandarin grafted on 12 rootstocks. Scientia Horticulturae, v.123, p.318-322, 2010.

COMIOTTO, A.; FACHINELLO, J.C.; HOFFMANN, A.; MACHADO, N.P.; GALARÇA, S.P.; BETEMPS, D.L. Vigor, floração, produção e qualidade de pêssegos 'Chimarrita' e 'Maciel' em função de diferentes porta-enxertos. Ciência Rural, v.42, p.788-794, 2012.

CRUZ, C.D. Programa Genes: versão windows. Viçosa: UFV, 2001. 642p.

CRUZ, C.D.; REGAZZI, A.J.; CARNEIRO, P.C.S. Modelos biométricos aplicados ao melhoramento genético. 3.ed. Viçosa: UFV, 2004. 480p.

FACHINELLO, J.C.; COUTINHO, E.F.; MARODIN, G.B.; BOTTON, M.; DE MIO, L.L.M. Normas técnicas e documentos de acompanhamento da produção integrada de pêssego. Pelotas: Universidade Federal de Pelotas, 2003. 92p.

FACHINELLO, J.C.; PASA, M. da S.; SCHMTIZ, J.D.; BETEMPS, D.L. Situação e perspectivas da fruticultura de clima temperado no Brasil. Revista Brasileira de Fruticultura, v.33, p.109-120, 2011. 
GONÇALVES, B.; MOUTINHO-PEREIRA, J.; SANTOS, A.; SILVA, A.P.; BACELAR, E.; CORREIA, C.; ROSA, E. Scion-rootstock interaction affects the physiology and fruit quality of sweet cherry. Tree Physiology, v.26, p.93-104, 2006.

HERNÁNDEZ, F.C.A.; PINOCHET, J.; MORENO, M.A.; MARTÍNEZ, J.J.; LEGUA, P. Performance of Prunus rootstocks for apricot in Mediterranean conditions. Scientia Horticulturae, v.124, p.354-359, 2010.

INSTITUTO BRASILEIRO DE GEOGRAFIA E ESTATÍSTICA. Produção agrícola municipal 2010. Disponível em: $<$ http://www. ibge.gov.br/home/estatistica/economia/pam/2010/default.shtm>. Acesso em: 9 jan. 2012.

MEDEIROS, C.A.B.; RASEIRA, M. do C.B. A cultura do pessegueiro. Brasília: Embrapa SPI; Pelotas: Embrapa-CPACT, 1998. 350p.

NAVA, G.A.; MARODIN, G.A.B.; SANTOS, R.P. dos. Reprodução do pessegueiro: efeito genético, ambiental e de manejo das plantas. Revista Brasileira de Fruticultura, v.31, p.1218-1233, 2009.

PICOLOTTO, L.; MANICA-BERTO, R.; PAZIN, D.; PASA, M. da S.; SCHMITZ, J.D.; PREZOTTO, M.E.; BETEMPS, B.; BIANCHI, V.J.; FACHINELLO, J.C. Características vegetativas, fenológicas e produtivas do pessegueiro cultivar Chimarrita enxertado em diferentes porta-enxertos. Pesquisa Agropecuária Brasileira, v.44, p.583-589, 2009.

PROTAS, J.F. da S.; MADAIL, J.C.M. Sistema de produção de pêssego de mesa na região da Serra Gaúcha. Bento Gonçalves: Embrapa Uva e Vinho, 2003. (Embrapa Uva e Vinho. Sistemas de produção, 3).
RATO, A.E.; AGUlHEIRO, A.C.; BARROSO, J.M.; RIQUELME, F. Soil and rootstock influence on fruit quality of plums (Prunus domestica L.). Scientia Horticulturae, v.118, p.218-222, 2008.

REMORINI, D.; TAVARINI, S.; DEGL'INNOCENTI, E.; LORETI, F.; MASSAI, R. GUIDI, L. Effect of rootstocks and harvesting time on the nutritional quality of peel and flesh of peach fruits. Food Chemistry, v.110, p.361-367, 2008.

ROCHA, M. da S.; BIANCHI, V.J.; FACHINELLO, J.C.; SCHMITZ, J.D.; PASA, M. da S.; SILVA, J.B. da. Comportamento agronômico inicial da cv. Chimarrita enxertada em cinco porta-enxertos de pessegueiro. Revista Brasileira de Fruticultura v.29, p.583-588, 2007.

ROSSI, A. de; FACHINELLO, J.C.; RUFATO, L.; PARISOTTO, E.; PICOLOTTO, L.; KRUGER, L.R. Comportamento do pessegueiro 'Granada' sobre diferentes porta-enxertos. Revista Brasileira de Fruticultura, v.26, p.446-449, 2004.

STRECK, E.V.; KÄMPF, N.; DALMOLIN, R.S.D.; KLAMT, E.; NASCIMENTO, P.C. do; SCHNEIDER, P.; GIASSON, E.; PINTO, L.F.S. Solos do Rio Grande do Sul. 2.ed. Porto Alegre: UFRGS, 2008. 222p.

TIMM, L.C.; REISSER JÚNIOR, C.; TAVARES, L.E.Q.; MADAIL, J.C.M.; MANKE, G.; LEMOS, F.D.; RADÜNZ, A.L.; LISBOA, H.; PRESTES, R.B.; MORO, M. Caracterização dos persicultores irrigantes e dos métodos de irrigação no pólo produtivo de pêssego da região de Pelotas. Revista Brasileira de Agrociência, v.13, p.413-417, 2007.

Recebido em 23 de abril de 2012 e aprovado em 10 de dezembro de 2012 